\title{
PROPHYLAXIS OF IMPLANT-RELATED INFECTIONS BY LOCAL RELEASE OF VANCOMYCIN FROM A HYDROGEL IN RABBITS
}

\author{
W. Boot ${ }^{1, *}$, H.C. Vogely ${ }^{1}$, P.G.J. Nikkels², B. Pouran ${ }^{1}$, M.H.P. van Rijen ${ }^{1}$, M.B. Ekkelenkamp ${ }^{3}$, G.M. Hänsch" \\ W.J.A. Dhert ${ }^{5}$ and D. Gawlitta ${ }^{6}$

\begin{abstract}
${ }^{1}$ Department of Orthopaedics, University Medical Centre Utrecht, Utrecht, the Netherlands
${ }^{2}$ Department of Pathology, University Medical Centre Utrecht, Utrecht, the Netherlands

${ }^{4}$ Department of Immunology, Heidelberg University, Heidelberg, Germany

${ }^{5}$ Faculty of Veterinary Medicine, Utrecht University, Utrecht, the Netherlands

${ }^{6}$ Department of Oral and Maxillofacial Surgery and Special Dental Care, University Medical Centre Utrecht, Utrecht, the Netherlands
\end{abstract} \\ ${ }^{3}$ Department of Medical Microbiology, University Medical Centre Utrecht, Utrecht, the Netherlands
}

\begin{abstract}
Local prophylaxis with antibiotic-loaded bone cement is a successful method to prevent post-operative infections in patients receiving orthopaedic implants. No comparable method is available for uncemented implants. Therefore, a hydrogel consisting of hyaluronic and polylactic acids was evaluated in a rabbit model for delivery of antimicrobial agents to prevent post-operative infections.

In a pilot study, the suitability of the in vivo model was assessed by testing the hydrogel as carrier material for antimicrobial agents. In the main study, the antimicrobial-agent-loaded hydrogel was evaluated for infection prophylaxis. Rabbits received a titanium rod intramedullary in the tibia after contamination with Staphylococcus aureus. The rods were coated with unloaded hydrogel (Gel), hydrogel loaded with $2 \%$ (Van2) or $5 \%$ vancomycin (Van5), bioactive glass (BAG) or N-acetyl-L-cysteine (NAC). To analyse the infection severity after $28 \mathrm{~d}$, histopathological, bacteriological, micro-computed tomographic and haematological analyses were performed.

In the pilot study, the Van5 group had less infection (0/6 infected) as compared to the Gel group (5/5, $p=0.000$ ) and the in vivo model was deemed suitable. In the main study, in the Van2 and Van5 groups, the number of infected animals was lower [1/6 $(p=0.006)$ and 2/6 $(p=0.044)$ infected, respectively]. In contrast, BAG and NAC groups showed no infection reduction (5/6 both groups, $p=0.997)$.

The hydrogel can be used as a local carrier of vancomycin for prophylaxis of implant-related infections. The present study showed promising results for local delivery of antibacterial agents by hydrogel to prevent implant-related infections.
\end{abstract}

Keywords: Implant-related infection, prophylaxis, in vivo, hydrogel, local delivery, vancomycin.

*Address for correspondence: Willemijn Boot, UMC Utrecht, Heidelberglaan 1003584 CX, Utrecht, the Netherlands.

Telephone number: +31 887556971 Email: w.boot@umcutrecht.nl

Copyright policy: This article is distributed in accordance with Creative Commons Attribution Licence (http://creativecommons.org/licenses/by-sa/4.0/).

\section{Introduction}

An orthopaedic-implant-related infection is a major complication that profoundly affects the patient's quality of life and is a burden for the health-care system. These infections are typically caused by biofilm-forming bacteria. The biofilm forms a microenvironment that supports the bacteria and protects them against both the host defence system and antimicrobial agents. As the metabolic activity of biofilm-residing bacteria decreases, so does their susceptibility to most antimicrobial agents (Costerton et al., 1995; Hoiby et al., 2010; Rochford et al., 2012) and higher antibiotic concentrations are required to inhibit their growth. Systemic antibiotic therapy, the standard for infection treatment and prophylaxis, 
may not yield sufficiently high concentrations at the implant site (Yeap et al., 2006). Local administration of antibiotics allows for much higher antibiotic penetration in biofilms and tissues. This approach has been effectively adopted in antibiotic-loaded cemented implants, which have been shown to lower infection rates and improve implant survival when compared to bone cement without antibiotics (Engesaeter et al., 2006; Engesaeter et al., 2003; Malchau et al., 2002).

In recent years, the number of implantations of uncemented prostheses has increased, especially in the young-patient population (Gee et al., 2013; Hailer et al., 2010; Kim et al., 2011). For uncemented nails, an antibiotic-coated nail is available (Moghaddam et al., 2019). However, hip and knee prostheses do lack the advantage of local infection prophylaxis that antibiotic-loaded bone cement offers. Various approaches have been tested to improve local infection prophylaxis in trauma and orthopaedic surgery (Uckay et al., 2013). A promising approach are antimicrobial delivery systems for local infection prophylaxis (ter Boo et al., 2015). For example, Neut et al. (2015) developed a biodegradable gentamicinhydroxyapatite coating on titanium alloy, covered with a protective, biodegradable poly(lactic-coglycolic acid) overlayer. This coating demonstrates infection prophylaxis for at least $7 \mathrm{~d}$ in a Staphylococcus aureus (S. aureus)-infected rabbit model. Furthermore, ter Boo et al. (2016) tested a thermo-responsive hyaluronic acid-poly(N-isopropylacrylamide) hydrogel for infection prophylaxis. The hydrogel, loaded with gentamicin, effectively prevented infection in a rabbit model of contaminated fracture treated with plating osteosynthesis. Furthermore, antibacterial coatings on implants have the potential of decreasing healthcare costs (Trentinaglia et al., 2018).

Ideally, local prophylaxis would consist of an easily applicable product that can be combined with the implants currently used in the clinic and offer the opportunity to tailor an individual antibacterial treatment strategy. Furthermore, such a method should offer a controlled delivery of the loaded agent into the local environment of the implant, achieving high local concentrations of the antibacterial agent but avoiding systemic side-effects. In this light, a fastresorbable hydrogel coating, that can be loaded with various antibacterial agents, may offer protection against early bacterial colonisation of the implant in the period following surgery (Campoccia et al., 2010). The study hypothesis was that a hydrogel could be used as a versatile carrier for antimicrobial agents on uncemented implants for infection prophylaxis.

The commercially available, biodegradable Defensive Antibacterial Coating ${ }^{\circledR}\left(\mathrm{DAC}^{\circledR}\right)$, consisting of hyaluronic acid and polylactic acid, was designed as a resorbable antibiotic-loaded hydrogel-coating for implants such as joint prostheses for protection against early post-operative infections. Release studies showed that $2 \%(\mathrm{w} / \mathrm{v})$ vancomycin-loaded
$\mathrm{DAC}^{\circledR}$ releases over $80 \%$ of the antibiotic within the first $24 \mathrm{~h}$ of in vitro incubation (Giavaresi et al., 2014), demonstrating that $\mathrm{DAC}^{\circledR}$ can facilitate a fast delivery of the loaded agent. Furthermore, the hydrogel, when coated on titanium implants, has no effect on the volume or timing of bone growth near the implant and does not induce an inflammatory reaction in a rabbit implant-model (Boot et al., 2017). Also, the hydrogel loaded with antibiotics (e.g. gentamicin and vancomycin) has proven to provide reduced rates of surgical site infections after internal osteosynthesis of closed fractures, hip or knee joint replacement and two-stage cement-less hip revision in three clinical studies (Malizos et al., 2017; Romano et al., 2016; Zagra et al., 2019).

The purpose of the present study was to investigate the hyaluronic-acid-based hydrogel DAC ${ }^{\circledR}$ as carrier for local delivery of antimicrobial agents for infection in an in vivo implant-related infection model. The hydrogel was loaded with vancomycin, a commonly used antibiotic in the orthopaedic field (van de Belt et al., 2001). Furthermore, in light of the emerging bacterial resistance to antibiotics (Raad et al., 1998) and the decreased sensitivity to antibiotics of biofilm-residing bacteria (Stewart and Costerton, 2001; Trampuz and Widmer, 2006), two non-antibiotic agents were tested. Namely, bioactive glass (BAG), which is currently used for treatment of chronic osteomyelitis in long bones (Drago et al., 2013b; Romano et al., 2014; Valimaki and Aro, 2006) and has osteoconductive (Lindfors and Aho, 2000), antibacterial (Lepparanta et al., 2008; Munukka et al., 2008) and antibiofilm properties (Drago et al., 2014b), and N-acetyl-L-cysteine (NAC), which has antibacterial properties (Drago et al., 2013a; Romano et al., 2013) and reduces biofilm formation in vitro (Drago et al., 2014a).

\section{Materials and Methods}

\section{Experimental design}

The present study comprised a pilot study and a larger main study. The pilot study was performed to assess the suitability of the in vivo model, to test whether the hydrogel could act as local carrier for agents to prevent infection. Further, the pilot study was performed to confirm that the hydrogel had no antibacterial effect in vivo, as shown in vitro (Drago et al., 2014a). In the animal experiment following the pilot, the hydrogel was evaluated as a carrier material for local delivery of antimicrobial agents for infection prophylaxis.

\section{Animal model and study timeline}

In both studies, an established rabbit implant-related infection model was used (Vogely et al., 2000). All animals received a sand-blasted titanium rod in the medullary canal of the left tibia at day 0 . To induce a local infection, $10^{5}$ colony forming units (CFUs) of $S$. aureus were inoculated in the medullary canal prior 
Table 1. Experimental timeframe of events.

\begin{tabular}{|c|c|}
\hline Action & Time point in study (day) \\
\hline Implantation & 0 \\
\hline X-ray imaging & 0 (postoperative) \\
\hline Blood drawings & 0 (postoperative), $7,14,21,28$ \\
\hline Fluorochrome injections & 3 and 10 or 7 and 21 \\
\hline Euthanasia & 28 \\
\hline
\end{tabular}

Table 2. Experimental and control groups included in the pilot and and follow-up animal studies.

\begin{tabular}{|c|c|c|c|c|}
\hline & Group name & Hydrogel & Hydrogel loaded with & Number \\
\hline \multirow{4}{*}{$\begin{array}{c}\text { Pilot animal } \\
\text { experiment }\end{array}$} & No gel & No & - & 6 \\
\cline { 2 - 5 } & Gel & Yes & - & 6 \\
\cline { 2 - 5 } & Van5 & Yes & $5 \%$ vancomycin & 6 \\
\hline \multirow{4}{*}{$\begin{array}{c}\text { Follow-up animal } \\
\text { experiment }\end{array}$} & No gel & No & - & 6 \\
\cline { 2 - 5 } & Gel & Yes & & 12 \\
\cline { 2 - 5 } & Van2 & Yes & $2 \%$ vancomycin & 6 \\
\cline { 2 - 5 } & Van5 & Yes & $5 \%$ vancomycin & 6 \\
\cline { 2 - 5 } & NAG & Yes & $10 \%$ bioactive glass & 6 \\
\hline
\end{tabular}

to implantation of the rod. Blood was taken weekly for analysis of neutrophil counts and erythrocyte sedimentation rates (ESRs). Also, fluorochromes were injected to label calcium incorporation at various time points. After $28 \mathrm{~d}$, euthanasia took place followed by histopathological and bacteriological analyses, microcomputed tomography $(\mu \mathrm{CT})$, as well as histological analysis of fluorochrome deposition (see also Table 1 for an overview of events).

\section{Pilot animal study}

First, the pilot study with 18 animals was performed according to the timeline described above, except for the fluorochrome injections. To assess whether the in vivo model system could be used to show a prophylactic effect for infection of a local carrier on implants, a group with $5 \%(\mathrm{w} / \mathrm{v})$ vancomycin mixed in the hydrogel was included. Further, to confirm that the hydrogel itself had no effect on the infection, a group with non-loaded hydrogel was included (see also Table 2 for an overview of the groups). As a negative control, a group without hydrogel coating was included. Power calculations were performed to estimate the required group size, taking the histological scores as the main outcome parameter. The calculation was based on the outcomes of previous, similar studies (Moojen et al., 2009; Vogely et al., 2000). With group sizes of 6 animals per group, the power to detect a difference of $25 \%$ in histological scoring for infection and inflammation was $79 \%$.

\section{Main animal study}

An animal study with 42 rabbits was performed. In the main study, the hydrogel was evaluated as a carrier for local delivery of antimicrobial agents for infection prophylaxis. As a positive control for prevention of infection, a group with $5 \%(\mathrm{w} / \mathrm{v})$ vancomycin loaded in the hydrogel was used. The negative control consisted of a group with no hydrogel coating. To determine whether a lower concentration of vancomycin could also reduce infection, a group with $2 \%(\mathrm{w} / \mathrm{v})$ vancomycin-loaded hydrogel was included. Also, the prophylactic efficacy of two alternative antibiotics was assessed. First, BAG has been proven to reduce bacterial growth in vitro (Drago et al., 2013b). Second, NAC has been shown to inhibit biofilm formation in vitro (Drago et al., 2014a). For the main animal experiment, a group size of 6 was used, except for the Gel group. As this latter group would be used for multiple comparisons with the other groups, it included 12 animals, increasing the likelihood of a representative outcome and, thus, decreasing the chance of a potential type I error. All experimental groups and the negative control were compared with the nonloaded hydrogel group. As it was not the study goal to find the optimal prophylactic agent, the test groups were not compared with each other.

\section{Animals, welfare and housing}

All experimental procedures were conducted with permission of the local Ethics Committee for Animal Experimentation at Utrecht University, Utrecht, the Netherlands (application number 2013.III.11.083). 16 week-old female New Zealand White rabbits (Charles River) were allowed to acclimatise for 7 to $14 \mathrm{~d}$ prior to surgery. They were housed in pairs at all times, except for the post-operative 2-3 d, until the surgical wounds were closed. Rabbits received $100 \mathrm{~g}$ of food (Stanrab, SDS, Essex, England) per day 
and water was available ad libitum. A weight loss exceeding $15 \%$ of the animal's initial weight, sepsis or shock were considered as exclusion criteria.

\section{Bacteria}

S. aureus Wood 46 (ATCC 10832), a strain used in previous studies with this animal model (Vogely et al., 2000), was used to induce a local infection in the tibiae.

For preparation of the stock suspension, a colony was grown overnight in Luria-Bertani medium at $37^{\circ} \mathrm{C}$. Subsequently, bacteria were grown until the logarithmic phase before being aliquoted and stored at $-80{ }^{\circ} \mathrm{C}$ in $8.5 \%$ glycerol until further use. The same stock was used throughout the study and each aliquot was cultured prior to the animal studies to assess the number of CFU/mL after thawing. The day before surgery, an aliquot of the stock was diluted with sterile phosphate-buffered saline (PBS) to obtain an inoculation dose of $50 \mu \mathrm{L}$ bacterial suspension containing a total of $10^{5} \mathrm{CFUs}$.

\section{Implants and hydrogel}

Cylindrical sand-blasted titanium implants (diameter $4 \mathrm{~mm}$, length $25 \mathrm{~mm}$ ), with an average surface roughness of $5.6 \mu \mathrm{m}$, were implanted (Adler Ortho SRL, Milan, Italy) (Fig. 1, left panel). Prior to implantation, implants were coated with the hydrogel [DAC ${ }^{\circledR}$, Novagenit ${ }^{\circledR}$, Mezzolombardo, Italy; patent number WO2010/086421 A1; CE-mark certified by notified body number 0426 as 349-00-00-DM and 350-00-00-DM]. DAC ${ }^{\circledR}$ consists of covalently linked hyaluronic acid and polylactic acid. The hydrogel was provided as a sterile powder in a syringe $(60 \mathrm{mg})$ and was reconstituted during surgery by mixing it with $1 \mathrm{~mL}$ sterile demineralised water using the connector provided (Fig. 1, middle panel), resulting in a hydrogel with a concentration of $6 \%(\mathrm{w} / \mathrm{v}) \mathrm{DAC}^{\circledR}$.

\section{Antimicrobials}

All antimicrobial agents were mixed with demineralised water prior to the preparation of the hydrogel. In the experimental groups, DAC ${ }^{\circledR}$ was loaded with 2 or $5 \%(\mathrm{w} / \mathrm{v})$ vancomycin (Hospira
Benelux BVBA, Brussels, Belgium) (Van2 and Van5 groups, respectively) (Table 2). BAG powder (S53P4, particle size $<45 \mu \mathrm{m}$, BonAlive Biomaterials Ltd, Turku, Finland) was loaded into the hydrogel with a weight/volume percentage of $10 \%$. Earlier research on BAG has proven that BAG can reduce bacterial growth in vitro (Drago et al., 2013b). NAC (A9165, Sigma-Aldrich) was loaded into the hydrogel with a weight/volume percentage of $0.5 \%$. This concentration was proven effective in vitro in a previous study for the reduction of $S$. epidermidis and S. aureus biofilm formation (Drago et al., 2014a).

\section{Surgery, analgesia and anaesthesia}

Surgery was performed using injection anaesthesia and under aseptic conditions. Rabbits received preoperative subcutaneously administered analgesia in the form of $0.03 \mathrm{mg} / \mathrm{kg}$ buprenorphine hydrochloride (Temgesic $^{\circledR}$, RB Pharmaceuticals Limited, Slough, UK). Anaesthesia was initiated by a subcutaneous injection of $10-15 \mathrm{mg} / \mathrm{kg}$ ketamine $\left(\right.$ Narketan $^{\circledR} 10$, Vétoquinol BV, 's-Hertogenbosch, the Netherlands) and $0.15-0.25 \mathrm{mg} / \mathrm{kg}$ dexdomitor (Orion Corporation, Espoo, Finland). Anaesthesia was maintained by an intravenous line of dexdomitor and ketamine $1: 10$ diluted with saline. To protect the eyes against dehydration, eye ointment was used (Added Pharma BV, Oss, the Netherlands). During surgery, rabbits received additional oxygen $(100 \%)$ through a mask.

The left paw was shaven and disinfected with $10 \%$ povidone-iodine (Betadine ${ }^{\circledR}$ solution, Meda Pharma B.B., Amstelveen, the Netherlands). After applying sterile draping, the knee joint was opened after making a medial-parapatellar incision. Anterior to the anterior-crucial ligament on the tibia, the medullary canal was opened with an awl and reamed by hand using a $4.1 \mathrm{~mm}$ diameter drill. Subsequently, a $50 \mu \mathrm{L}$ bacterial suspension containing $10^{5} \mathrm{CFU}$ S. aureus was injected under otherwise sterile conditions into the cavity using a pipette. Next, the implant, with or without coating, was inserted into the medullary canal. The joint capsule was closed with Vicryl size 3-0 sutures (Ethicon, Johnson \& Johnson) and the skin with Monocryl size 3-0 sutures (Ethicon,
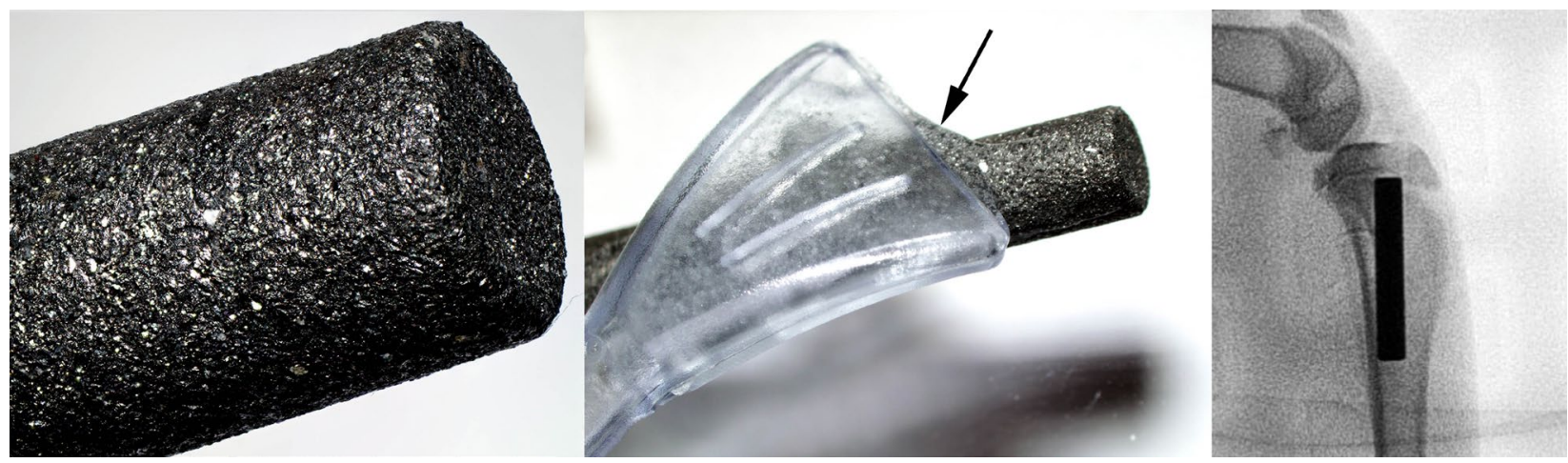

Fig. 1. Surface, coating and implantation of the titanium rod. Left panel: close-up of the rough surface of the titanium rod. Central panel: coating of the titanium rod with transparent hydrogel (arrow) using a spreader. Right panel: post-operative X-ray image of the rabbit left tibia, showing correct positioning of the titanium implant in the proximal medullary tibial cavity. Diameter of the rod: $4 \mathrm{~mm}$. 
Johnson \& Johnson). X-ray imaging was performed to verify the position of the implants in the proximal medullary tibial cavity, with an undamaged cortex (Fig. 1, right panel). Anaesthesia was reversed using 0.5-1.0 mg/kg Atipam (Eurovet Animal Health B.V., Bladel, the Netherlands). Post-operative analgesia with subcutaneously administered $0.03 \mathrm{mg} / \mathrm{kg}$ buprenorphine hydrochloride was applied every $8 \mathrm{~h}$ for the first $48 \mathrm{~h}$.

\section{Euthanasia}

Rabbits were sacrificed 28 d after surgery with an overdose of sodium pentobarbital (Euthanimal $40 \%$, Alfasan Nederland BV, Woerden, the Netherlands) administered intravenously under general anaesthesia.

\section{Post-mortem sample acquisition and analyses}

After sacrificing the animals, the skin of both paws was shaven, disinfected with $10 \%$ povidone-iodine and covered with sterile drapes. After removing skin and soft tissues, the anterior parts of the proximal tibiae were extracted under aseptic conditions, using a rotary saw with a saw blade (Dremel, model 300, and Dremel Cut-Off Wheel No. 409, Breda, the Netherlands). The bone pieces were placed into a sterile tube, weighed and stored on ice to prevent further bacterial growth. This procedure was performed for both tibiae of all animals. The posterior parts of the left tibiae with the implant were sawed off and fixed in formalin.

\section{Bacteriology of anterior tibiae}

The anterior bone fragments of the tibiae were weighed and homogenised in $10 \mathrm{~mL}$ PBS using a tissue homogeniser (Polytron PT3100; Kinetica Benelux, Best, the Netherlands) at 2,500 rpm for $3 \mathrm{~min}$ and subsequently at 6,000 rpm for $5 \mathrm{~min}$. Then, serial 10-fold dilutions from these homogenates were plated onto $5 \%$ sheep blood agar plates (Becton Dickinson, Alphen aan den Rijn, the Netherlands). After an overnight incubation at $37^{\circ} \mathrm{C}$, the S. aureus CFUs on the plates were counted. For each tibia, the number of CFUs of bacteria/g of bone was calculated.

\section{Bone-implant contact}

Before the embedding process, the bone volume fraction percentage was measured within $180 \mu \mathrm{m}$ from the implant surface using a $\mu \mathrm{CT}$ scanner (Quantum FX, Perkin Elmer) to analyse the bone apposition on the implant surface. Data were acquired under a tube voltage of $90 \mathrm{kV}$, a tube current of $180 \mu \mathrm{A}$, a scan time of $3 \mathrm{~min}$ per sample and a voxel size of $60 \times 60 \times 60 \mu^{3}$. Then, images were reconstructed automatically in three dimensions (3D) using the built-in $\mu \mathrm{CT}$ software (Analyze 11.0). The same software was used to convert the $3 \mathrm{D}$ images into 512 2D slices in tiff format. To calculate the amount of bone at the implant surface, the images were first thresholded globally. Multiple regions of interest (ROIs) were generated to measure the bone- implant contact. The implant position in the bone was used to generate ROI (Fig. 5b). To eliminate the partial volume effects due to the rough surface of the implant, $\mathrm{ROI}_{1}$ was expanded by two pixels $\left(\mathrm{ROI}_{2}\right)$. The region representing the bone-implant contact was defined as the volume sandwiched between $\mathrm{ROI}_{2}$ and the area created by radially enlarging $\mathrm{ROI}_{2}$ with 3 pixels $\left(\mathrm{ROI}_{3}\right)$. Then, the bone in $\mathrm{ROI}_{3}$ was locally segmented and the bone volume within $\mathrm{ROI}_{3}$ was calculated. The amount of bone volume when compared with the total volume of $\mathrm{ROI}_{3}$ yielded the percentage of bone-implant contact. The surface of the implant directly adjacent to the removed anterior part of the tibia was excluded from the analysis.

\section{Histopathology}

The posterior part of the left tibia, containing the implant, was embedded in methyl methacrylate (MMA). Briefly, the fixed samples were dehydrated through a graded ethanol series and embedded in MMA solution $[0.8 \mathrm{~mL} / \mathrm{mL}$ methyl methacrylate (Merck KGaA), $28 \mathrm{mg} / \mathrm{mL}$ benzoyl peroxide (513474, Sigma-Aldrich) and $0.2 \mathrm{~mL} / \mathrm{mL}$ Plastoid-N (nonylphenyl-polyethyleneglycol acetate, SigmaAldrich Chemie $\mathrm{GmbH}$ )]. After MMA polymerisation, 20-30 $\mu$ m-thick sections were made along the transverse axis of the tibia using a sawing microtome (Leica SP1600). MMA sections were stained with $0.3 \%$ basic fuchsin solution and subsequently with $1 \%$ methylene blue solution. For semi-quantitative scoring of infection, sections were scored for signs of infection and inflammation according to the scoring system by Vogely et al. (2000). The scoring system quantifies different parameters for infection and inflammation (cortical destruction, enlargement of Haversian canals, periosteal thickening, presence of leukocytes, micro abscesses, granulation tissue and fibrosis in the cortex and medullary canal), resulting in a score between 0 (no infection) and 56 (serious infection). One proximal and one distal section of each tibia, including the implant, were blindly examined together by a pathologist using a light microscope.

\section{Haematology}

Blood samples were taken from an ear vein and sent to the Laboratory of Clinical Chemistry and Haematology at the UMC Utrecht for measuring ESR and neutrophil counts pre-operatively and 1, 2, 3 and 4 weeks post-operatively.

\section{Fluorochromes}

To visualise dynamic bone formation during the experimental period, rabbits were injected with fluorochromes (van Gaalen et al., 2010). In each experimental group, half of the rabbits were injected on day 3 and 10 and the other half were injected on day 7 and 21 with xylenol orange (xylenol orange tetrasodium salt; 398187, Sigma-Aldrich) and calcein green (calcein disodium salt, 21030, Fluka analytical, Sigma-Aldrich), respectively. Unstained MMA 
sections were analysed for presence of fluorochromes in the bone directly adjacent to the implant to determine the time of bone deposition around the implant. A score of 1 was given if the label was present. A score of 0 was given if no bone or no label was present. The scores of all samples in a group were averaged per time point, resulting in a score between 0 (bone deposition in none of the animals) and 1 (bone deposition in all animals).

\section{Statistical analyses}

In the pilot experiment, the No gel and Van5 groups were compared to the Gel group using a one-way ANOVA with Dunnett's post-hoc test. In the main study, for evaluating the efficacy of the hydrogel as a carrier, No gel, Van2, Van5, BAG and NAC groups were compared to the Gel group using a one-way ANOVA with Dunnett's post-hoc test for all analyses and time points. All statistical calculations were

a
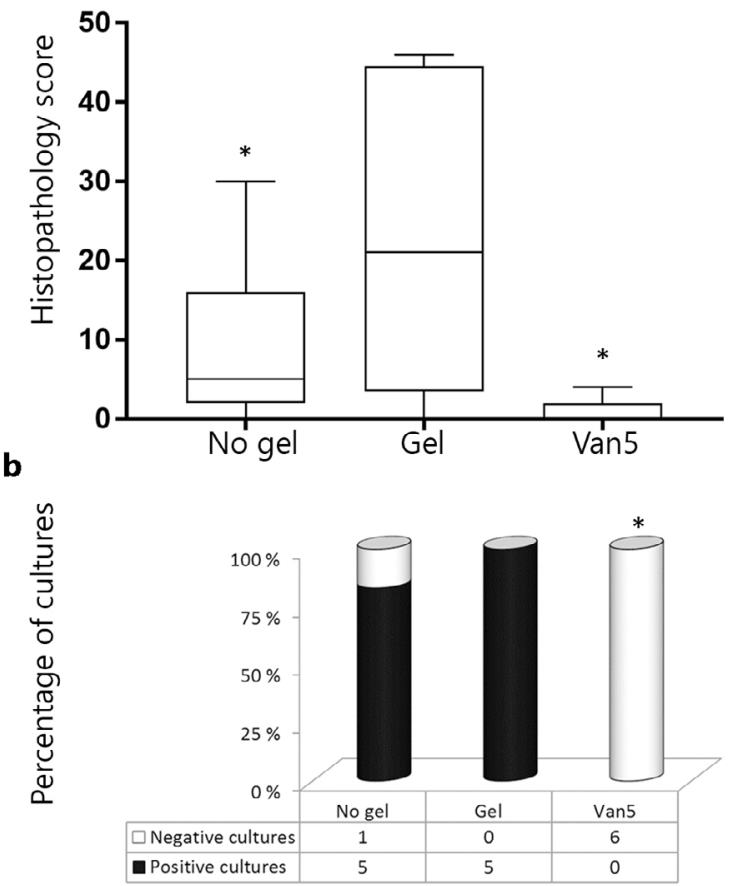

C

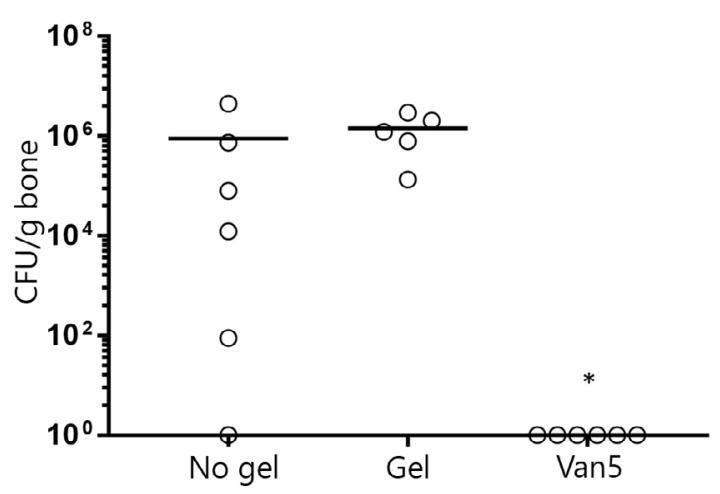

performed in SPSS (Microsoft Windows version 20.0; SPSS, Inc., Chicago, Illinois). A $p<0.05$ was considered statistically significant.

\section{Results}

\section{Pilot study}

General

In the pilot study, one rabbit from the Gel group died of an unknown cause on day 3 and was replaced. However, the replacement animal also died, with strong suspicion of sepsis due to infection.

\section{Histopathology}

The semiquantitative scoring of the histological sections showed that the Van5 group had a significantly lower infection grade when compared with the Gel group (mean score $1 \pm 1.6, p=0.000$ ) (Fig.

d
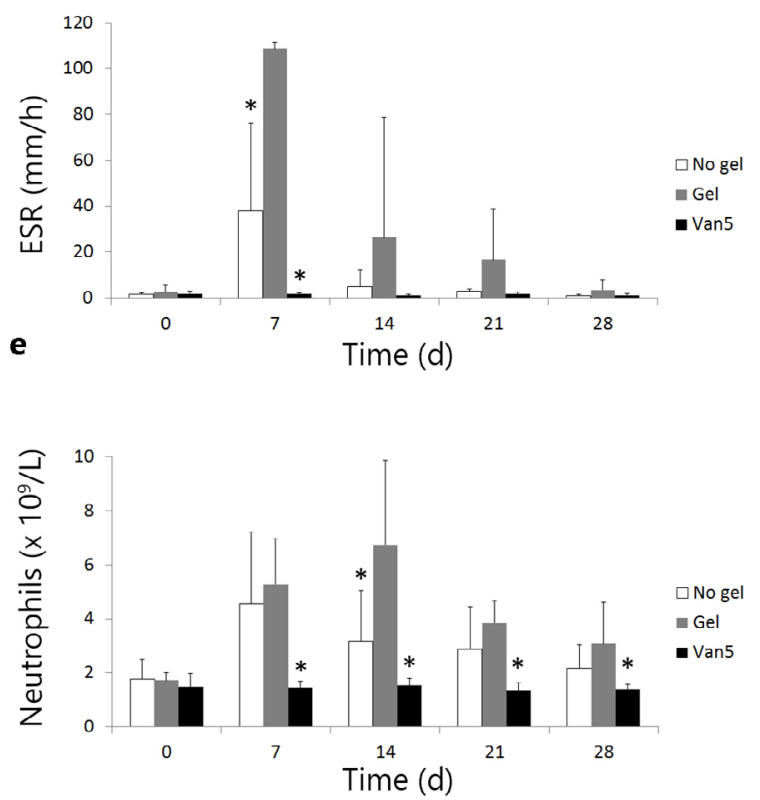

Fig. 2. Results of the pilot study. (a) Semiquantitative infection grading in the pilot study. (b) Number of rabbits with a positive culture in the anterior tibia after $28 \mathrm{~d}$ in the pilot study. (c) Number of CFU/g of bone from the left tibiae. (d) ESRs. (e) Neutrophil counts. ${ }^{*}$ indicates a $p<0.05$, compared to the Gel group. 
2a). Also, lower infection and inflammation scores were seen in the No gel group when compared to the Gel group (mean scores $9.5 \pm 10.3$ and $22.2 \pm 18.9$ respectively, $p=0.034)$.

\section{Bacteriology}

In 5 out of 6 rabbits in the No gel group and in 5 out of 5 rabbits in the Gel group, bacteria were found in the bone of the anterior tibiae $(p=0.445)$ (Fig. 2b). In contrast, none of the rabbits were positive for bacteria in the Van5 group ( $p=0.000$, compared to Gel group), indicating that no local infection was present at day 28 . Bacterial culture resulted in average $[ \pm$ standard deviation (SD)] CFU counts of $1.1 \times 10^{6}\left( \pm 1.1 \times 10^{6}\right)$ for the No gel group and $1.4 \times 10^{6}\left( \pm 1.8 \times 10^{6}\right)$ for the Gel group $(p=0.699)$, whereas the Van5 group was culture negative ( $p=0.131$, compared to the Gel group) (Fig. $2 c)$. None of the cultures from the contralateral side was positive.

\section{Haematology}

The rabbits in the Van5 group showed lower neutrophil counts than those in the Gel group at day $7,14,21$ and $28(p=0.007,0.001,0.003$ and 0.024, respectively) (Fig. $2 \mathrm{~d}$ ). The No gel group demonstrated lower neutrophil counts at day 14 as compared to the Gel group $(p=0.020)$ (Fig. $2 \mathrm{~d}$ ). The No gel and Van 5 groups exhibited lower ESR values as compared to the Gel group at day 7 ( $p=0.000$ for both groups) (Fig. 2e).

\section{Main study}

\section{General}

All rabbits recovered well from the surgical intervention. X-ray imaging showed correct positioning of the implant in the left tibiae. During the study period, two rabbits died. One rabbit of the BAG group died at day 7. Autopsy showed a strong indication for septicaemia. This animal was replaced. One rabbit of the Gel group was found deceased at day 12. Autopsy was not conclusive in defining the cause of death. This animal was replaced; however, the replacement animal also died, possibly

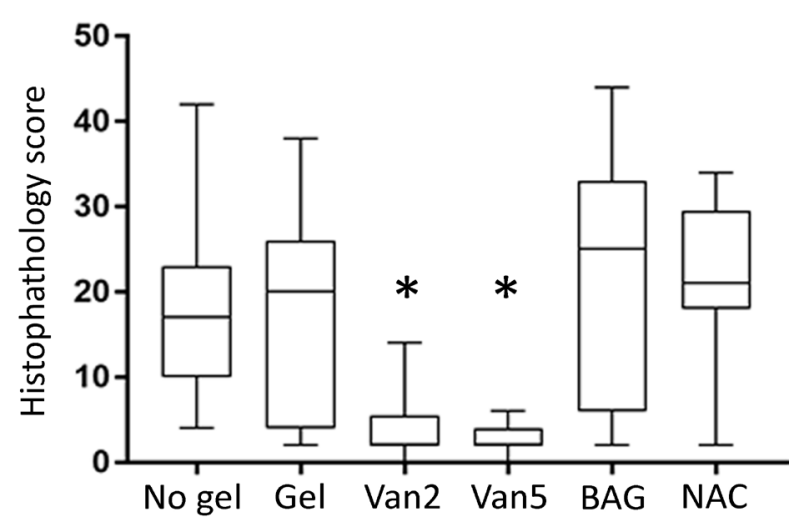

Fig. 3. Histopathological scores are shown, indicating the severity of infection and inflammation for all groups. * indicates a $p<0.05$, compared to the Gel group. of septicaemia. Results obtained from the deceased rabbits were excluded from the study, leaving the Gel group with 11 animals instead of 12.

\section{Histopathology}

The histopathology data of the Gel group were analysed using the Kolmogorov-Smirnov test, which showed a normal data distribution $(p=0.263)$. Microscopical analysis of the histological slides showed similar infection scores in the Gel group as compared to the No gel group (mean scores $17.7 \pm 11.9$ and $17.5 \pm 9.9$, respectively, $p=0.955$ ) (Fig. $3)$. The average histopathology scores of the rabbits in both vancomycin groups were lower when compared to the Gel group (mean score $3.8 \pm 3.9, p=0.001$ for the Van2 group and mean score $2.7 \pm 1.5, p=0.000$ for the Van 5 group). No differences were found between the BAG or NAC groups when compared to the Gel group (mean scores $22.2 \pm 14.6, p=0.575$ and $21.8 \pm 8.8$, $p=0.641$, respectively).

\section{Bacteriology}

Bone samples from the anterior tibiae of both left (contaminated) and right (control) rabbit paws were cultured to detect for presence and number of bacteria (Fig. 4). All cultures of the right-control tibiae were negative. In the No gel and Gel groups, 2 out of 6 and 1 of 11 rabbits ( $p=0.720$ ), respectively, did not have infection-positive cultures after $28 \mathrm{~d}$. This suggested that some rabbits cleared the infection themselves (Fig. 4a). In the Van2 and Van5 groups,
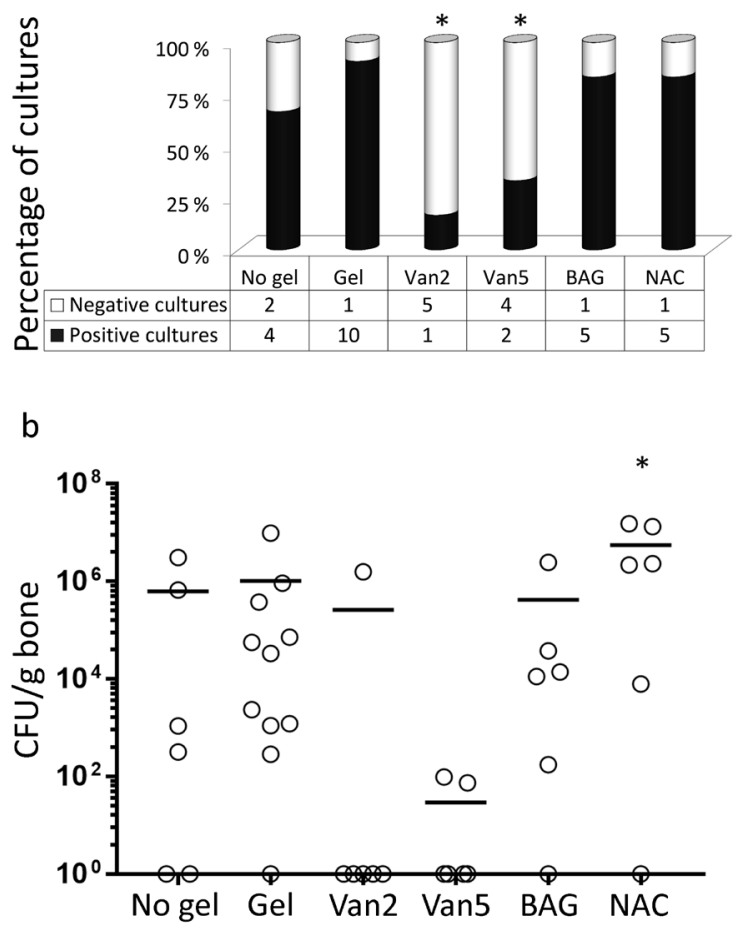

Fig. 4. Bacteriological results. (a) Percentages of rabbits with negative and positive cultures in the bone samples. (b) Quantitative culture results of the bacterial cultures are shown in CFU/g of bone. 
more rabbits had negative bacterial cultures, i.e. 5 out of $6(p=0.006)$ and 4 out of $6(p=0.044)$, respectively. The BAG and NAC groups demonstrated similar results to the Gel control group, as in both groups 1 out of $6(p=0.997)$ rabbits had a negative culture. Bacterial culture resulted in average $( \pm S D)$ CFU counts of $4.2 \times 10^{5}\left( \pm 9.9 \times 10^{5}\right)$ and $6.3 \times 10^{5}\left( \pm 1.2 \times 10^{6}\right)$ $(p=1.000)$ for the No gel and Gel groups, respectively (Fig. 4b). For the Van2 and Van5 groups, average CFU counts were $2.6 \times 10^{5}\left( \pm 6.4 \times 10^{5}\right)(p=0.989)$ and $2.9 \times 10^{1}\left( \pm 4.5 \times 10^{1}\right)(p=0.962)$, respectively. The BAG group had a similar average of $4.2 \times 10^{5}\left( \pm 9.9 \times 10^{5}\right)$ $(p=0.996)$ as compared to the Gel control group. The NAC group had a higher bacterial count of $6.6 \times 10^{6}$ $\left( \pm 6.9 \times 10^{6}\right)(p=0.033)$, compared to the Gel group.

\section{$\mu \mathrm{CT}$}

Infection induced destruction of the original cortex and increase in bone volume at the periosteal side, as could be observed in the images from the Gel, BAG and NAC groups (Fig. 5a), whereas the No gel group showed mild periosteal bone formation. Both groups with vancomycin exhibited an intact cortex and no increases in periosteal bone. All groups exhibited an average percentage of bone-implant contact of 40-60 \% (Fig. 5c). The Gel group showed a similar average amount of bone-implant contact (42.4\%) to the No gel group $(45.4 \%, p=0.376)$. The Van 2 and Van 5 groups had higher average percentages of boneimplant contact (54.4\% and $52.8 \%$, respectively), although the difference was only significant for the Van2 group ( $p=0.009$ and $p=0.210$, respectively). The BAG and NAC groups demonstrated similar bone-implant contact percentages $(38.5 \%$ and $39.7 \%$, respectively) as compared to the Gel group ( $p=0.681$ and $p=0.598$, respectively).

\section{Haematology}

The Gel group demonstrated blood values similar to the No gel group at all time points. The Van2 and Van 5 groups showed lower ESR ( $p=0.015$ and $p=0.004$, respectively) (Fig. 6a) and neutrophil values
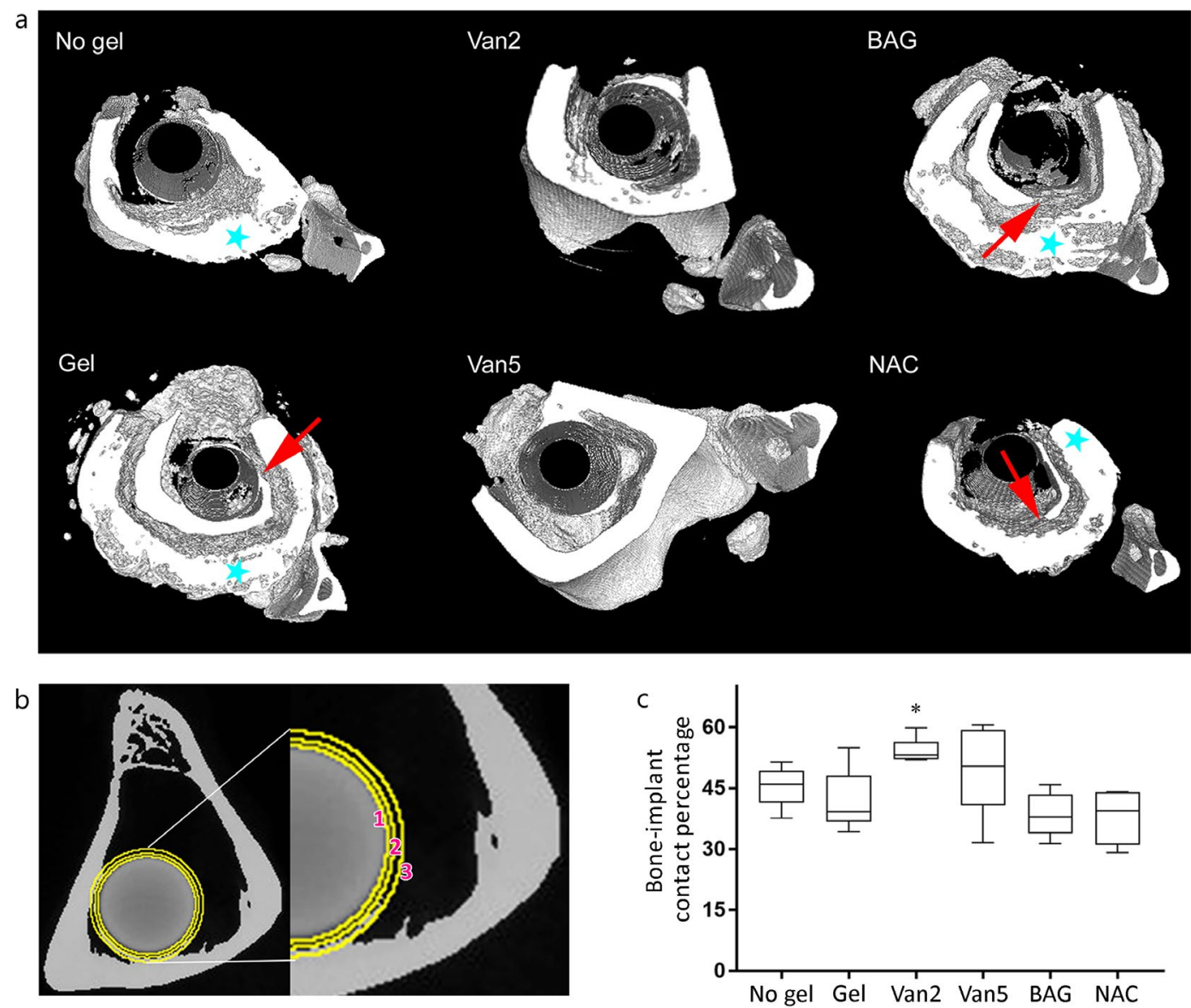

Fig. 5. Infection effect on bone architecture and bone-implant contact. (a) Overview of representative 3D $\mu \mathrm{CT}$ images of the left tibia showing destruction of the cortex (red arrows) and visual growth of periosteal bone (blue stars) The titanium rod is removed from the images allowing for visualisation of the bone on the implant surface. The anterior tibia was removed for bacteriology and is missing from these images. (b) ROIs were generated to enable measuring the bone-implant contact. The implant was detected by global thresholding and defined $\mathrm{ROI}_{1}$ (inner yellow circle 1). ROI $\mathrm{R}_{1}$ was radially expanded 2 pixels, which resulted in $\mathrm{ROI}_{2}$ (middle yellow circle, 2). Then, $\mathrm{ROI}_{2}$ was enlarged with 3 pixels (outer yellow circle, 3 ). The enlarged region was defined as $\mathrm{ROI}_{3}$ and represented the region of bone-implant contact. (c) Percentages of boneimplant contact measured at the implant surface. ${ }^{*}$ indicates a significant difference when compared to the Gel group $(p<0.05)$. 
( $p=0.001$ and $p=0.000$, respectively) (Fig. 6b) at day 7 when compared with the Gel group. The Van 5 group also exhibited decreased neutrophil counts at day 28 as compared with the Gel group $(p=0.041)$. The blood values of the BAG and NAC groups did not differ from the Gel group at any time point.

\section{Histological analysis of fluorochrome deposition}

In Fig. 7, images taken by light and fluorescence microscopy are shown to visualise the presence or absence of fluorochromes at different time points (days 3, 7, 10 and 21). Except for 1 rabbit in the Gel group, no rabbits presented bone apposition around the implant at day 3 (Table 3). At day 7, the Van2 group exhibited increased bone growth around the implant as compared to the Gel group ( $p=0.031$ ). By day 21, the Van5 group was the only group that showed bone apposition in all animals. In general, all groups exhibited an increased incidence of bone deposition around the implant over time.

\section{Discussion}

The present study demonstrated that $\mathrm{DAC}^{\circledR}$ could successfully deliver vancomycin for local prophylaxis against implant-related $S$. aureus infections in a rabbit model. DAC ${ }^{\circledR}$ loaded with $2 \%$ or $5 \%$ vancomycin reduced the infection severity and incidence in an in vivo model of implant-related infection. Furthermore, the vancomycin-loaded hydrogel coating improved
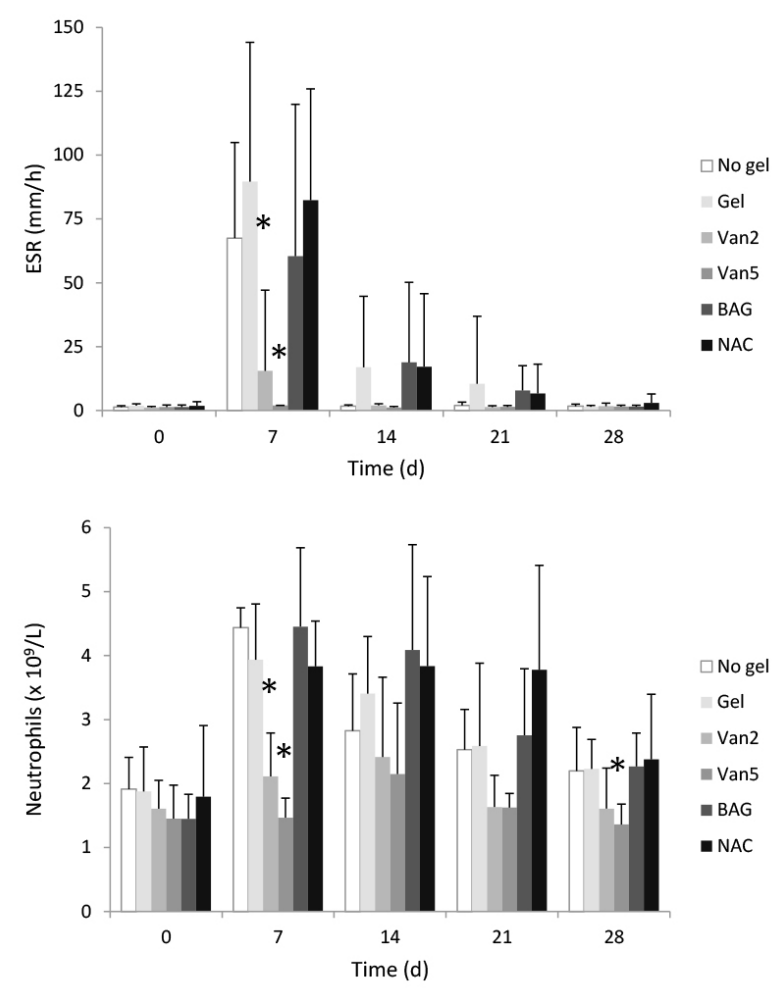

Fig. 6. Rabbit blood values measured during the experimental period. (a) ESRs. (b) Neutrophil counts. * indicates a significant difference when compared to the Gel group for each time point $(p<0.05)$. bone-implant contact and demonstrated lower scores for infection, inflammation, ESR and neutrophil counts at day 7 as compared to the unloaded hydrogel. Moreover, the Van2 group exhibited a higher bone-implant contact percentage and a higher incidence of bone deposition around the implant than the Gel group. This trend was also observed for the Van5 group, although the differences were not significant. The BAG and NAC groups did not show reductions in any of the infection parameters as compared to those of the Gel group.

The pilot study was designed to test whether the group with $5 \%$ vancomycin loaded in the hydrogel was suitable as a positive control group and to confirm that the hydrogel itself did not exert an antibacterial activity in vivo. Indeed, the Van5 group demonstrated a reduced infection as compared to the Gel group, demonstrating that applying it to an implant could prevent infection in vivo and that it could serve in the following animal experiment as a positive control.

As $\mathrm{DAC}^{\circledR}$ alone does not exert an antibacterial activity in vitro (Drago et al., 2014a), the No gel and Gel groups in the pilot experiment were expected to demonstrate similar outcomes. Surprisingly, the differences in the histological scoring and blood values between the No gel and the Gel group in the pilot study suggested that the presence of the hydrogel could lead to a more severe infection. Because this finding was unexpected, a group without hydrogel coating was included in the following animal experiment to investigate whether this effect could be reproduced. However, in the subsequent experiment, no significant differences could be demonstrated between the group without hydrogel and the group with empty hydrogel coating for any of the tested parameters. Therefore, there was no conclusive evidence that the hydrogel caused a more severe infection when applied without antibacterial agents.

Loading the hydrogel with vancomycin did not eliminate the infection in all rabbits, as some bacterial cultures in the Van groups were positive. Histological scores for infection and inflammation were lower when using vancomycin-loaded hydrogel, indicating a mitigation of the infection severity. Also, the animals did not receive systemic antibiotics in support of the local antibacterial prophylaxis, as routinely applied in the clinics. This finding suggested that local antibiotics alone were not enough to prevent infection in the described model. Interestingly, the results indicated that the Van2 group performed better than the Van5 group, suggesting that $2 \%$ vancomycin could be a sufficient dose for infection prophylaxis. However, the study was not designed to find an optimal dose of vancomycin to be loaded in the hydrogel and more research should be conducted to find more conclusive evidence in this context.

DAC was specifically designed as a coatingmaterial for uncemented bone implants. Therefore, it would be interesting to further functionalise 
the hydrogel with osteoconductive materials that can stimulate bone growth towards the implant (Fassbender et al., 2014; Rammelt et al., 2006; Stadlinger et al., 2008). Bone-implant contact, which provides stability to the implant, is essential for the long-term clinical success of uncemented implants (Matassi et al., 2013). In the present study, bone-implant contact was already observed by fluorochrome incorporation at day 3. Furthermore, in most groups, more than half of the samples exhibited bone-implant contact at day 21. One of the antibacterial materials incorporated, BAG, is also well-known for its bone regenerative potential (Valimaki and Aro, 2006). Nevertheless, due to the persistence of infection, which induces bone resorption (Del Pozo and Patel, 2009; Parvizi et al., 2012), the osteoconductive effects could not be studied for the BAG group.

BAG has also been shown to exert an antibacterial effect (Lepparanta et al., 2008; Munukka et al., 2008), which is thought to reside in the simultaneous increase in $\mathrm{pH}$ value and the rise in osmotic pressure caused by the release of ions, causing an unfavourable environment for bacteria (Stoor et al., 1998). Although BAG has been shown to inhibit bacterial growth in vitro at similar concentrations of various aerobic bacteria, including S. aureus (Munukka et al., 2008), loading DAC ${ }^{\circledR}$ with BAG did not prevent infection in any of the animals. It may be possible that an

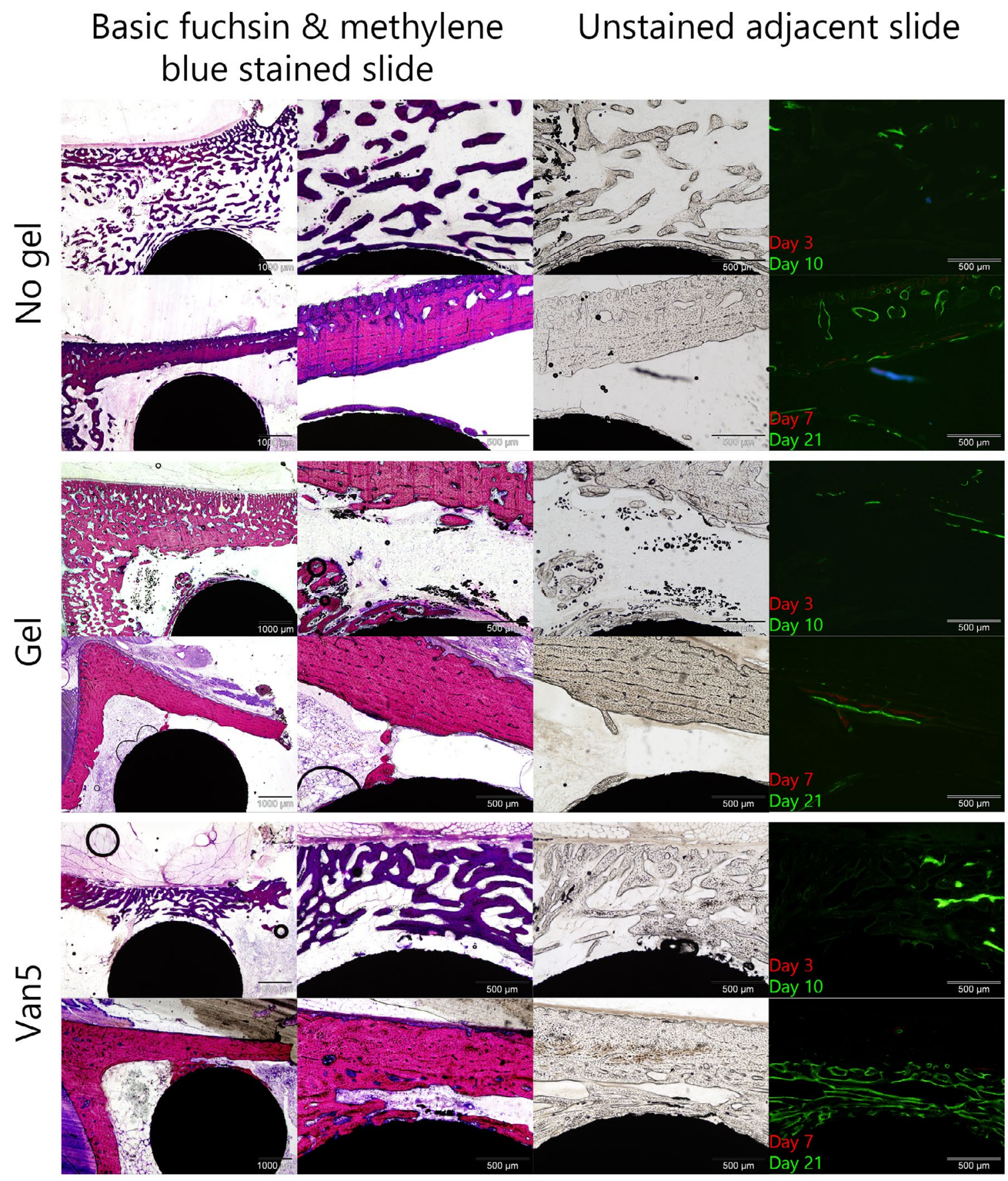

Fig. 7. Images of stained and unstained MMA slides. Images of the three left columns were acquired by light microscopy. Images in the right column were acquired by fluorescence photography to visualise the presence or absence of fluorochromes at different time points. 
Table 3. Incidence of bone deposition around the implant. A score of 0 indicates bone deposition in none of the animals, a score of 1 indicates bone deposition in all animals. * indicates a significant difference when compared to the Gel group $(p<0.05)$.

\begin{tabular}{|c|c|c|c|c|}
\hline & Day 3 & Day 7 & Day 10 & Day 21 \\
\hline No gel & 0.00 & 0.17 & 0.50 & 0.83 \\
\hline Gel & 0.08 & 0.10 & 0.42 & 0.60 \\
\hline Van2 & 0.00 & $0.67^{*}$ & 0.50 & 0.83 \\
\hline Van5 & 0.00 & 0.17 & 0.50 & 1.00 \\
\hline BAG & 0.00 & 0.00 & 0.50 & 0.33 \\
\hline NAC & 0.00 & 0.33 & 0.00 & 0.50 \\
\hline
\end{tabular}

insufficient absolute amount of BAG was locally available to facilitate an antibacterial effect, however, more research needs to be performed to confirm this theory. Similarly, applying a NAC-loaded hydrogel on the implant did not reduce the infection in the rabbit model. The concentration of NAC used in the current study was proven to be effective in an in vitro setting for reduction of biofilm formation by S. epidermidis and S. aureus (Drago et al., 2014a). In congruence with the amount of BAG, the total amount of NAC might also have been too small to prevent infection in this model.

A hydrogel provides flexibility in the choice of the functional agent. A previous study has shown that the hydrogel used in the present study can be loaded with a range of antimicrobial agents and can be applied on various implant surfaces (Drago et al., 2014a). Considering the risks of bacteria developing antibiotic resistance that is associated with the use of antibiotics (Campoccia et al., 2006), the application of alternatives has become very attractive. Different types of antibacterial agents may be combined for a more effective infection prophylaxis. Hydrogels can be rapidly reconstituted and easily applied on virtually every type of uncemented orthopaedic implant during the surgical procedure, eliminating the need for a variety of prosthetic implants with specific coatings (Goodman et al., 2013).

In conclusion, locally administered vancomycinloaded $\mathrm{DAC}^{\circledR}$ reduced infection rates in an animal model of implant-related infections. The use of antimicrobial agents such as BAG and NAC did not exert a prophylactic effect.

\section{Acknowledgements}

The authors thank Dr Albert Huisman for his valuable advice on the haematological parameters and Marc Jansen for his assistance with the microbial work.

The study was performed under the multicentre Collaborative Project "I.D.A.C.", funded by the European Commission, within the $7^{\text {th }}$ Framework Programme on Research Technological Development and Demonstration, grant number 277988. WB was partially supported by Biomet.

\section{References}

Boot W, Gawlitta D, Nikkels PGJ, Pouran B, van Rijen MHP, Dhert WJA, Vogely HC (2017) Hyaluronic acid-based hydrogel coating does not affect bone apposition at the implant surface in a rabbit model. Clin Orthop Relat Res 475: 1911-1919.

Campoccia D, Montanaro L, Arciola CR (2006) The significance of infection related to orthopedic devices and issues of antibiotic resistance. Biomaterials 27: 2331-2339.

Campoccia D, Montanaro L, Speziale P, Arciola CR (2010) Antibiotic-loaded biomaterials and the risks for the spread of antibiotic resistance following their prophylactic and therapeutic clinical use. Biomaterials 31: 6363-6377.

Costerton JW, Lewandowski Z, Caldwell DE, Korber DR, Lappin-Scott HM (1995) Microbial biofilms. Annu Rev Microbiol 49: 711-745.

Del Pozo JL, Patel R (2009) Clinical practice. Infection associated with prosthetic joints. N Engl J Med 361: 787-794.

Drago L, Boot W, Dimas K, Malizos K, Hänsch GM, Stuyck J, Gawlitta D, Romanò CL (2014a) Does implant coating with antibacterial-loaded hydrogel reduce bacterial colonization and biofilm formation in vitro? Clin Orthop Relat Res 472: 3311-3323.

Drago L, De Vecchi E, Mattina R, Romano CL (2013a) Activity of N-acetyl-L-cysteine against biofilm of Staphylococcus aureus and Pseudomonas aeruginosa on orthopedic prosthetic materials. Int J Artif Organs 36: 39-46.

Drago L, Romano D, De Vecchi E, Vassena C, Logoluso N, Mattina R, Romanò CL (2013b) Bioactive glass BAG-S53P4 for the adjunctive treatment of chronic osteomyelitis of the long bones: an in vitro and prospective clinical study. BMC Infect Dis 13: 584. DOI: 10.1186/1471-2334-13-584.

Drago L, Vassena C, Fenu S, De Vecchi E, Signori V, De Francesco R, Romanò CL (2014b) In vitro antibiofilm activity of bioactive glass S53P4. Future Microbiol 9: 593-601.

Engesaeter LB, Espehaug B, Lie SA, Furnes O, Havelin LI (2006) Does cement increase the risk of infection in primary total hip arthroplasty? Revision rates in 56,275 cemented and uncemented primary 
THAs followed for 0-16 years in the Norwegian Arthroplasty Register. Acta Orthop 77: 351-358.

Engesaeter LB, Lie SA, Espehaug B, Furnes O, Vollset SE, Havelin LI (2003) Antibiotic prophylaxis in total hip arthroplasty: effects of antibiotic prophylaxis systemically and in bone cement on the revision rate of 22,170 primary hip replacements followed 0-14 years in the Norwegian Arthroplasty Register. Acta Orthop Scand 74: 644-651.

Fassbender M, Minkwitz S, Strobel C, Schmidmaier G, Wildemann B (2014) Stimulation of bone healing by sustained bone morphogenetic protein 2 (BMP-2) delivery. Int J Mol Sci 15: 8539-8552.

Gee MJ, Ajuied A, Shah Z, George M, Bankes MJ (2013) Systematic review of total hip arthroplasty in patients under 30 years old. Hip Int 23: 345-351.

Giavaresi G, Meani E, Sartori M, Ferrari A, Bellini D, Sacchetta AC, Meraner J, Sambri A, Vocale C, Sambri V, Fini M, Romanò CL (2014) Efficacy of antibacterial-loaded coating in an in vivo model of acutely highly contaminated implant. Int Orthop 38: 1505-1512.

Goodman SB, Yao Z, Keeney M, Yang F (2013) The future of biologic coatings for orthopaedic implants. Biomaterials 34: 3174-3183.

Hailer NP, Garellick G, Karrholm J (2010) Uncemented and cemented primary total hip arthroplasty in the Swedish Hip Arthroplasty Register. Acta Orthop 81: 34-41.

Hoiby N, Bjarnsholt T, Givskov M, Molin S, Ciofu $\mathrm{O}$ (2010) Antibiotic resistance of bacterial biofilms. Int J Antimicrob Agents 35: 322-332.

Kim YH, Kim JS, Park JW, Joo JH (2011) Comparison of total hip replacement with and without cement in patients younger than 50 years of age: the results at 18 years. J Bone Joint Surg Br 93: 449-455.

Lepparanta O, Vaahtio M, Peltola T, Zhang D, Hupa L, Hupa M, Ylänen H, Salonen JI, Viljanen MK, Eerola E (2008) Antibacterial effect of bioactive glasses on clinically important anaerobic bacteria in vitro. J Mater Sci Mater Med 19: 547-551.

Lindfors NC, Aho AJ (2000) Tissue response to bioactive glass and autogenous bone in the rabbit spine. Eur Spine J 9: 30-35.

Malchau H, Herberts P, Eisler T, Garellick G, Soderman P (2002) The swedish total hip replacement register. J Bone Joint Surg Am 84-A Suppl 2: 2-20.

Malizos K, Blauth M, Danita A, Capuano N, Mezzoprete R, Logoluso N, Drago L, Romanò CL (2017) Fast-resorbable antibiotic-loaded hydrogel coating to reduce post-surgical infection after internal osteosynthesis: a multicenter randomized controlled trial. J Orthop Traumatol 18: 159-169.

Matassi F, Botti A, Sirleo L, Carulli C, Innocenti M (2013) Porous metal for orthopedics implants. Clin Cases Miner Bone Metab 10: 111-115.

Moghaddam A, Weis J, Haubruck P, Raven TF, Addali A, Schmidmaier G (2019) Evaluation of the clinical use of the ETN PROtect(R) in non-union therapy. Injury 50 Suppl 3: 32-39.
Moojen DJ, Vogely HC, Fleer A, Nikkels PG, Higham PA, Verbout AJ, Castelein RM, Dhert WJ (2009) Prophylaxis of infection and effects on osseointegration using a tobramycin-periapatite coating on titanium implants - an experimental study in the rabbit. J Orthop Res 27: 710-716.

Munukka E, Lepparanta O, Korkeamaki M, Vaahtio M, Peltola T, Zhang D, Hupa L, Ylänen H, Salonen JI, Viljanen MK, Eerola E (2008) Bactericidal effects of bioactive glasses on clinically important aerobic bacteria. J Mater Sci Mater Med 19: 27-32.

Neut D, Dijkstra RJ, Thompson JI, Kavanagh C, van der Mei HC, Busscher HJ (2015) A biodegradable gentamicin-hydroxyapatite-coating for infection prophylaxis in cementless hip prostheses. Eur Cell Mater 29: 42-55.

Parvizi J, Adeli B, Zmistowski B, Restrepo C, Greenwald AS (2012) Management of periprosthetic joint infection: the current knowledge: AAOS exhibit selection. J Bone Joint Surg Am 94: e104. DOI: 10.2106/ JBJS.K.01417.

Raad I, Alrahwan A, Rolston K(1998) Staphylococcus epidermidis: emerging resistance and need for alternative agents. Clin Infect Dis 26: 1182-1187.

Rammelt S, Illert T, Bierbaum S, Scharnweber D, Zwipp H, Schneiders W (2006) Coating of titanium implants with collagen, RGD peptide and chondroitin sulfate. Biomaterials 27: 5561-5571.

Rochford ET, Richards RG, Moriarty TF (2012) Influence of material on the development of deviceassociated infections. Clin Microbiol Infect 18: 11621167.

Romano CL, Logoluso N, Meani E, Romanò D, De Vecchi E, Vassena C, Drago L (2014) A comparative study of the use of bioactive glass S53P4 and antibiotic-loaded calcium-based bone substitutes in the treatment of chronic osteomyelitis: a retrospective comparative study. Bone Joint J 96-B: 845-850.

Romano CL, Malizos K, Capuano N, Mezzoprete R, D'Arienzo M, Van Der Straeten C, Scarponi S, Drago L (2016) Does an antibiotic-loaded hydrogel coating reduce early post-surgical infection after joint arthroplasty? J Bone Jt Infect 1: 34-41.

Romano CL, Toscano M, Romano D, Drago L (2013) Antibiofilm agents and implant-related infections in orthopaedics: where are we? J Chemother 25: 67-80.

Stadlinger B, Pilling E, Mai R, Bierbaum S, Berhardt R, Scharnweber D, Eckelt U (2008) Effect of biological implant surface coatings on bone formation, applying collagen, proteoglycans, glycosaminoglycans and growth factors. J Mater Sci Mater Med 19: 1043-1049.

Stewart PS, Costerton JW (2001) Antibiotic resistance of bacteria in biofilms. Lancet 358: 135-138.

Stoor P, Soderling E, Salonen JI (1998) Antibacterial effects of a bioactive glass paste on oral microorganisms. Acta Odontol Scand 56: 161-165.

ter Boo GA, Arens D, Metsemakers WJ, Zeiter S, Richards RG, Grijpma DW, Eglin D, Moriarty TF (2016) Injectable gentamicin-loaded thermoresponsive hyaluronic acid derivative prevents infection in a rabbit model. Acta Biomater 43: 185-194. 
ter Boo GJ, Grijpma DW, Moriarty TF, Richards RG, Eglin D (2015) Antimicrobial delivery systems for local infection prophylaxis in orthopedic- and trauma surgery. Biomaterials 52: 113-125.

Trampuz A, Widmer AF (2006) Infections associated with orthopedic implants. Curr Opin Infect Dis 19: 349-356.

Trentinaglia MT, Van Der Straeten C, Morelli I, Logoluso N, Drago L, Romano CL (2018) Economic evaluation of antibacterial coatings on healthcare costs in first year following total joint arthroplasty. J Arthroplasty 33: 1656-1662.

Uckay I, Hoffmeyer P, Lew D, Pittet D (2013) Prevention of surgical site infections in orthopaedic surgery and bone trauma: state-of-the-art update. J Hosp Infect 84: 5-12.

Valimaki VV, Aro HT (2006) Molecular basis for action of bioactive glasses as bone graft substitute. Scand J Surg 95: 95-102.

van de Belt $H$, Neut D, Schenk W, van Horn JR, van der Mei HC, Busscher HJ (2001) Infection of orthopedic implants and the use of antibiotic-loaded bone cements. A review. Acta Orthop Scand 72: 557571.

van Gaalen SM, Kruyt MC, Geuze RE, de Bruijn JD, Alblas J, Dhert WJ (2010) Use of fluorochrome labels in in vivo bone tissue engineering research. Tissue Eng Part B Rev 16: 209-217.

Vogely HC, Oosterbos CJ, Puts EW, Nijhof MW, Nikkels PG, Fleer A, Tonino AJ, Dhert WJ, Verbout AJ (2000) Effects of hydroxyapatite coating on Ti-6A1$4 \mathrm{~V}$ implant-site infection in a rabbit tibial model. J Orthop Res 18: 485-493.

Yeap JS, Lim JW, Vergis M, Au Yeung PS, Chiu CK, Singh H (2006) Prophylactic antibiotics in orthopaedic surgery: guidelines and practice. Med J Malaysia 61: 181-188.

Zagra L, Gallazzi E, Romano D, Scarponi S, Romano C (2019) Two-stage cementless hip revision for peri-prosthetic infection with an antibacterial hydrogel coating: results of a comparative series. Int Orthop 43: 111-115.

\section{Discussion with Reviewers}

Olivier Guillaume: One of the most important factors to consider during the development of local antibiotic therapies is the risk that bacteria become resistant to the treatment, even though high dosages of drugs are available locally (as it seems to be the case in the present study). Is the discovery of microorganisms surrounding antibiotic-loaded prostheses a really bad outcome? And what are consequently the next possible steps for practicians?

Authors: The presence of microorganisms at euthanasia proved that infection prophylaxis was not successful in some animals. However, there was a clear difference in the groups that did not receive local antibiotics, indicating that locally applied antibiotics could be a powerful method to prevent infection. Furthermore, the animals in this model did not receive systemic antibiotics, so this finding may suggest that a combination of local and systemic antibiotics is important for infection prophylaxis.

Sebastian AJ Zaat: In view of an antimicrobial activity, what is the degradation speed of the hydrogel? Will the hydrogel remain after having released its antimicrobial "payload" and will the hydrogel itself provide any remaining bacteria with a safe site, for instance, preventing them from being phagocytosed? Are they penetrating the hydrogel? Does it provide a site for biofilm formation?

Authors: We did not test whether the antibacterial agents were eluted from the hydrogel before the hydrogel broke down in vivo. Further experiments, specifically designed for this purpose, would be needed before being able to speculate about this topic.

Sebastian AJ Zaat: Will an empty hydrogel either in the presence or absence of infection have an influence on bone apposition? This is particularly relevant in case of incorporation of a bioglass. Will the bioglass be in contact with the rabbit bone or will it be shielded? Authors: The empty hydrogel has proven to have no influence on bone apposition in a non-infected animal model with an implant (Boot et al., 2017). Although the bioglass is mixed with the hydrogel, it could be possible that the ions released from the bioglass are also released from the hydrogel; however, this was not tested.

Reviewer: How can consistency be ensured in terms of volume and distribution of hydrogel along the implant? Is this a concern for clinical application? Authors: The volume of the hydrogel depends mainly on the surface area of the implant. The goal is to coat the complete implant surface to be as effective as possible.

Reviewer: Is the hydrogel excluded from the intramedullary space considering the implant was $4 \mathrm{~mm}$ and the hole in the bone was $4.1 \mathrm{~mm}$ ? I would suspect that much of the hydrogel was retained at the entry point of the bone.

Authors: During the coating procedure, the hydrogel was coated on the whole surface of the implant. There was some hydrogel retained indeed at the entry point of the bone, however, most was inserted into the intramedullary canal. This topic has been covered in a previous study (Drago et al., 2014a).

Editor's note: The Scientific Editor responsible for this paper was Fintan Moriarty. 